\title{
Cavitational Mechanisms in Ultrasound-accelerated Fibrinolysis
}

\author{
Adrian F. Prokop*, Azita Soltani*, Ronald A. Roy ${ }^{\dagger}$
}

*Pre-clinical Research Department, EKOS Corporation, Bothell, Washington, USA

${ }^{\dagger}$ Department of Aerospace and Mechanical Engineering, Boston University, Boston, Massachusetts, USA

Address all correspondence and reprint requests to:
Azita Soltani
Pre-clinical Research Department
EKOS Corporation
11911 N Creek Parkway S
Bothell, WA 98011, USA
Email: asoltani@ekoscorp.com
Phone: 425-415-3100
Fax: 425-415-3103

Running Title: US-accelerated fibrinolysis 


\title{
Cavitational Mechanisms in Ultrasound-accelerated Fibrinolysis
}

\begin{abstract}
The role of both inertial and stable cavitation was investigated during in vitro ultrasoundaccelerated fibrinolysis by recombinant tissue plasminogen activator (rt-PA) in the presence and absence of Optison $^{\mathrm{TM}}$. A unique treatment configuration applied ultrasound, rt-PA, and Optison ${ }^{\mathrm{TM}}$ to the interior of a plasma clot. Lysis efficacy was measured as clot weight reduction.

Cavitational mechanisms were investigated by monitoring subharmonic and broadband noise. In the absence of Optison ${ }^{\mathrm{TM}}$, 1.7 MHz, pulsed ultrasound with 1.5 MPa peak-negative pressure applied for $30 \mathrm{~min}$ resulted in $45 \pm 19 \%$ lysis enhancement relative to rt-PA alone. Cavitation was not detected, indicating a role of non-cavitational effects of ultrasound. The addition of Optison ${ }^{\mathrm{TM}}$ increased lysis enhancement to $88 \pm 25 \%$. Inertial cavitation was present only at the start of the exposure, while low-amplitude subharmonic emissions persisted throughout. Additional protocols suggested a possible correlation between the increased lysis in the presence of Optison ${ }^{\mathrm{TM}}$ and the subharmonic emission, indicating a potentially important role of stable rather than inertial cavitation in microbubble-enhanced ultrasound-accelerated rt-PA-mediated thrombolysis.
\end{abstract}

Keywords: Fibrinolysis, Optison ${ }^{\mathrm{TM}}$, Tissue Plasminogen Activator, Passive Cavitation Detection, Broadband Noise, Subharmonic Emission. 


\section{INTRODUCTION}

Ultrasound of frequency greater than $0.5 \mathrm{MHz}$ has been shown to accelerate clot dissolution by fibrinolytic agents in vitro (Blinc et al. 1993; Francis et al. 1992; Harpaz et al. 1993; Lauer et al. 1992) and in vivo (Kashyap et al. 1994; Kornowski et al. 1994; Riggs et al. 1997). Although the mechanisms are not fully understood, it is speculated that ultrasound accelerates enzymatic fibrinolysis primarily through non-thermal mechanisms by increasing transport of drug molecules into the clot (Francis et al. 1995). Mechanical effects of ultrasound such as streaming, radiation force, and cavitation have the ability to influence drug transport. Acoustic cavitation is generally acknowledged as playing a significant role in ultrasoundaccelerated fibrinolysis (Everbach and Francis 2000). Indeed, the addition of ultrasound contrast agents has been shown to increase the effectiveness of ultrasound-accelerated enzymatic fibrinolysis (Cintas et al. 2004; Mizushige et al. 1999; Tachibana and Tachibana 1995; Wu et al. 1998). While it is recognized that the presence of cavitation increases lysis, the specific types of bubble activity most beneficial to enzymatic fibrinolysis is still unclear. Mechanisms related to both inertial cavitation (i.e. intense localized stresses, microjets) and stable cavitation (i.e. cavitation microstreaming, bubble translation) may be responsible for the enhanced drug transport and lysis.

In an ongoing effort to elucidate the mechanisms of ultrasound-accelerated enzymatic fibrinolysis, the current study was performed to investigate the role of both inertial and stable cavitation during in vitro fibrinolysis in the presence and absence of ultrasound contrast agent. The ultrasound-tipped catheter (EKOS Corporation, Bothell, WA, USA) used in this study is intended for direct infusion of drug into a thrombus, with simultaneous local ultrasound exposure. Previous tests have demonstrated that the ultrasound catheter enhances enzymatic fibrinolysis in the presence of a fibrinolytic agent, as verified by an increase in concentration of the fibrin degradation product D-Dimer (Soltani 2005). The ultrasound generated by this catheter in the 
absence of lytic agent resulted in no clot weight loss, suggesting that the ultrasound does not cause clot disruption.

A particular motivation for the current study was to use a unique in vitro treatment configuration in which the ultrasound, fibrinolytic agent, and contrast agent were applied to the interior of the clot rather than at the external surface. The typical in vitro setup for ultrasoundaccelerated fibrinolysis experiments consists of external applications of either or both the ultrasound and lytic agents (i.e. clot submerged in fluid medium containing drug/microbubbles, and exposed to external ultrasound). Using similar setups, prior catheter-based ultrasound fibrinolysis studies (Shlansky-Goldberg et al. 1996; Tachibana and Tachibana 1995, 1997) placed the transducer adjacent to rather than within the clot, with drug surrounding the clot. In the external exposure setup, the ultrasound-drug and ultrasound-microbubble interaction sites are at the thrombus surface. It is probable that the dominant mechanisms for ultrasound-accelerated fibrinolysis vary depending on the location where ultrasound, drug, and contrast agent interact. For example, the effect of bulk acoustic streaming on drug distribution may be less important within the dense fibrin matrix of the clot than it would be in the fluid surrounding the clot. In addition, the viscoelastic properties of the surrounding medium can affect the behavior of the microbubbles in response to the ultrasound (Yang 2005). The current setup presented a more pertinent model for catheter-based ultrasound-accelerated fibrinolysis in which the lytic agents are injected directly into the clot which is internally exposed to ultrasound (Soltani 2005). 


\section{MATERIALS AND METHODS}

\section{Ultrasound Source}

Catheter-mounted ultrasound transducers (EKOS Corporation) were used. The device consisted of a tiny piezoelectric ceramic cylinder mounted coaxially along the distal tip of the catheter. The dimensions of the radiating cylindrical surface were $0.9 \mathrm{~mm}$ outer diameter by 2.1 mm length. The catheter transducer was driven at its standard clinical operating parameters, for which safety has previously been confirmed in vivo in the absence of contrast agent (Soltani 2005). A function generator (33120A; Agilent, Palo Alto, CA, USA) connected to a RF power amplifier (2100L; ENI Technology Inc., Rochester, NY, USA) was used to drive the transducers at $1.7 \mathrm{MHz}$ in pulsed mode with a pulse duration on the order of 1000 cycles. Based on a measurement of total radiated acoustic power, the spatial-average temporal-average intensity at the surface of the transducer was $4.9 \mathrm{~W} / \mathrm{cm}^{2}$. The spatial-peak peak-rarefaction pressure was measured using a needle hydrophone to be $1.5 \mathrm{MPa}$. Due to geometric spreading of the field, acoustic pressure was greatest at the surface of the transducer, and decreased with radial distance away from the transducer.

To control transducer heating due to imperfect electric-to-acoustic energy conversion, the catheter was fitted within a thin-walled polyimide sleeve (1.07 mm ID, $0.025 \mathrm{~mm}$ wall thickness) within which degassed water was circulated. Catheter temperature was monitored by a miniature T-type thermocouple attached to the transducer surface. During treatment, the rate of water flowing through the catheter lumen and into the surrounding polyimide sleeve was adjusted to limit the transducer surface temperature to $43^{\circ} \mathrm{C}$, the upper limit for transducer-patient contact surface temperature as specified by international standards for medical ultrasound (IEC 2004). In clinical practice a water-filled polyimide sleeve is not used, but rather the catheter surface temperature is controlled using a software algorithm to proportionally scale back the driving power when necessary. For the current in vitro study, however, it was of interest to drive the catheters at maximum power (i.e. not scaled back to compensate for temperature rise) for the 
duration of the treatment. In the presence of the polyimide sleeve, the spatial-peak pressure amplitude in the clot volume was estimated to be $10 \%$ less than the pressure at the transducer surface due to geometric beam divergence over the transducer-clot separation distance (attenuation was found to be negligible). This reduction, being less than the hydrophone measurement error for peak pressure $(\sim 17 \%)$, was considered negligible, and so the spatial-peak pressure in the clot may be reasonably approximated by the spatial-peak pressure on the surface of the bare catheter.

\section{Clot Model}

Clots were formed from citrated human pooled plasma (Precision BioLogic, Dartmouth, Nova Scotia, Canada). $2.0 \mathrm{~mL}$ frozen plasma was thawed and then heated at $37^{\circ} \mathrm{C}$ in an opentopped test tube for 20 min prior to clot formation to allow the plasma to reach its equilibrium gas saturation concentration at $37^{\circ} \mathrm{C}$. The plasma was transferred to a custom-made polyolefin test tube of $1.27 \mathrm{~cm}$ ID and $0.03 \mathrm{~cm}$ wall thickness. The tube material was chosen so that its acoustic impedance, determined to be 1.4 MRayl based on speed of sound and density measurement, was close to that of water to avoid standing waves in the test tube. For tests with ultrasound contrast agent, 9.2 $\mu \mathrm{L}$ Optison ${ }^{\mathrm{TM}}$ perflutren $\left(\mathrm{C}_{3} \mathrm{~F}_{8}\right)$ human albumin microsphere suspension (Amersham Health Inc., Princeton, NJ, USA) was gently mixed into the plasma for a concentration of $2.5 \times 10^{6}$ bubbles/mL plasma. This step ensured a consistent, uniform distribution of Optison ${ }^{\mathrm{TM}}$ throughout each clot. In clinical practice, catheter-delivered Optison ${ }^{\mathrm{TM}}$ could be less ideally distributed and confined to a more limited volume of the clot. The addition of Optison ${ }^{\mathrm{TM}}$ to plasma before clot formation was not expected to affect clot structure since the mean size of the microbubbles was on the order of reported pore sizes for in vitro plasma clot (Carr 1987). Concerning the stability of Optison $^{\mathrm{TM}}$ during clot formation, no significant degradation was expected since Optison ${ }^{\mathrm{TM}}$ microbubbles diluted in air-saturated media have been observed to persist for up to several hours (Podell 1999). Since individual Optison ${ }^{\mathrm{TM}}$ vials were used over several days (maximum 3 days), the vials were vented to $\mathrm{C}_{3} \mathrm{~F}_{8}$ reservoir during Optison ${ }^{\mathrm{TM}}$ removal to preserve the headspace gas 
composition. Clotting was initiated by adding $200 \mu \mathrm{L}$ each of $0.25 \mathrm{M}$ calcium chloride (SigmaAldrich, St. Louis, MO, USA) and $10 \mathrm{IU} / \mathrm{mL}$ bovine thrombin (VWR, West Chester, PA, USA). Immediately after addition of thrombin, the ultrasound catheter and two adjacent polyimide drug delivery lumens ( $0.404 \mathrm{~mm} \mathrm{ID}, 0.025 \mathrm{~mm}$ wall thickness), positioned on a $1.9 \mathrm{~mm}$ radius on opposite sides of the catheter, were simultaneously inserted vertically within the exposure tube to approximately $2 / 3$ of the depth below the top surface of clot (Fig. 1a). The clot was incubated for $10 \mathrm{~min}$ prior to treatment by submerging the polyolefin exposure tube in a $37^{\circ} \mathrm{C}$ water bath, where it remained for the duration of the treatment.

\section{Treatment Protocol}

Various combinations of drug, ultrasound, and Optison ${ }^{\mathrm{TM}}$ were applied to clots so that their individual and combined contributions to clot lysis and cavitation activity could be studied. Six treatment protocols were tested: 1) $C$-control; 2) $D-$ drug-only; 3) $D+U S-$ drug and ultrasound; 4) $D+U S+O P$ —drug, ultrasound, and Optison ${ }^{\mathrm{TM}}$; 5) $D+U S+O P$ (pre) —drug, ultrasound, and Optison ${ }^{\mathrm{TM}}$ that was pretreated with ultrasound; 6) $U S+O P$ - ultrasound and Optison $^{\mathrm{TM}}$.

For all treatment protocols, the clot remained in the $37^{\circ} \mathrm{C}$ water bath for a total of $45 \mathrm{~min}$. For the control (protocol 1), the catheter was inserted but ultrasound remained off. Previous tests (Soltani 2005) showed no additional change in control clot weight due to presence of drug delivery lumens or injected fluid (saline), and therefore were not included in the control protocol. In protocols where lytic drug was used (protocols 2-5), a 5 min bolus injection of 5,000 IU/mL recombinant tissue plasminogen activator, rt-PA (Activase ${ }^{\circledR}$; Genentech Inc., San Francisco, CA, USA), was delivered by syringe pump through each of the two drug delivery lumens, resulting in a total of $0.08 \mathrm{~mL}$ rt-PA delivered into the clot in the vicinity of the ultrasound transducer. For protocol 6, an equivalent volume of phosphate-buffered saline was delivered through the lumens instead. In protocols where ultrasound was applied (protocols 3-6), the 30 min exposure began immediately after completion of drug delivery (or at the equivalent time for protocol 6). For 
protocol 5, the Optison ${ }^{\mathrm{TM}}$ in the clot was pre-exposed to the catheter's ultrasound field just prior to drug delivery. The purpose of this treatment protocol was to investigate the effect of the inertial cavitation activity of Optison ${ }^{\mathrm{TM}}$ (observed a priori during preliminary testing) on fibrinolysis by temporally separating this activity from the rt-PA treatment. The catheter and drug-delivery lumens were inserted and clot incubation proceeded as normal. Before drug was delivered, however, the catheter was turned on for two brief ( $\sim 6 \mathrm{~s}$ each) ultrasound exposures at the same acoustic output as the 30 min exposure, which activated and subsequently eliminated the inertial cavitation activity of the Optison ${ }^{\mathrm{TM}}$.

Clot Lysis

Lysis efficacy was evaluated by measuring final clot weights. Upon completion of treatment, the clot was removed from the test tube, placed between two layered filter paper preparations, and pressed with $10 \mathrm{lbs}$ weight for $5 \mathrm{~min}$ to expel the serum. The remaining fibrin film was carefully removed from the filter paper and placed on a precision electronic balance, and the clot weight was recorded. For each treatment protocol, an average clot weight, $W_{i}(\mathrm{mg})$, was compiled from 10 independent samples. Percent clot lysis, $L_{i}(\%)$, was calculated for all treatment groups as the percent weight reduction relative to the control weight, $W_{C}$ :

$L_{i}(\%)=\left[\frac{W_{C}-W_{i}}{W_{C}}\right] \times 100$

The term "lysis" as used in this paper refers in general to a reduction in clot weight. Specific mechanisms for that reduction (i.e. enzymatic fibrinolysis, mechanical disruption) shall be inferred from the results of the current and previous studies.

For treatments with drug and ultrasound together, the lysis enhancement relative to drug alone, $L E_{i}(\%)$, was calculated as the percent increase in lysis relative to the drug-only lysis. Equation 2 shows equivalent expressions for $L E_{i}$ in terms of either the percent lysis or the clot weights, where $L_{D}$ and $W_{D}$ are the corresponding drug-only parameters: 
$L E_{i}(\%)=\left[\frac{L_{i}}{L_{D}}-1\right] \times 100=\left[\frac{W_{C}-W_{i}}{W_{C}-W_{D}}-1\right] \times 100$

Statistical analysis was performed on the raw clot weight data using One-Way ANOVA and Fisher's Least Significant Difference multiple comparison t-test at a 0.05 level of significance (SPSS 12 for Windows; SPSS Inc., Chicago, IL, USA).

Cavitation Detection and Analysis

A passive cavitation detection scheme was used to monitor cavitation generated within the clot during treatment. A broadband (less than $3 \mathrm{~dB}$ variation in response out to $10 \mathrm{MHz}$ ), weakly focused (1.3 cm diameter, $5.5 \mathrm{~cm}$ focal length), PVDF hydrophone (Sonic Concepts, Woodinville, WA, USA) was used to monitor acoustic emissions from the clot. The water surrounding the exposure tube was degassed to less than $36 \%$ of saturation and filtered to $0.2 \mu \mathrm{m}$ to ensure that cavitation would not occur anywhere outside the clot, a condition that is further ensured by the fact that geometric spreading of the ultrasound field resulted in a lower peak pressure in the water bath compared to the peak pressure in the clot. A micro-positioning stage was used to position the clot such that the hydrophone's focal zone was centered at a distance of $1.5 \mathrm{~mm}$ adjacent to the source transducer's central axis (Fig. 1b). This off-center positioning was used to reduce the direct signal level received from the source ultrasound, which increased the dynamic range available for cavitation detection. The hydrophone voltage signal passed through an anti-aliasing $10 \mathrm{MHz}$ lowpass filter, was digitized at $50 \mathrm{MHz}$ sampling rate using an 8 bit analog-to-digital converter board (NI-5911; National Instruments, Austin, TX, USA), and stored on a computer for offline analysis. The software used to control signal acquisition and to perform signal analysis was written in LabVIEW 7.1 (National Instruments, Austin, TX, USA).

To limit the collected data to manageable file sizes, "snapshots" of noise activity were obtained by digitizing transient capture "bursts" of the hydrophone signal at seven time points during the treatment: $0,1,2,4,8,16$, and 30 min. Each transient capture burst was timed to 
coincide with the exposure interval of the pulsed ultrasonic catheter. Each snapshot consisted of a digitized record of 100 consecutive bursts, corresponding to $3.33 \mathrm{~s}$ of elapsed treatment time.

Data analysis consisted of tracking and quantifying certain frequency components detected in the scattered acoustic emissions that are characteristic of cavitation activity. Subharmonic emission at half the driving frequency, a characteristic of stable nonlinear bubble oscillation (Eller and Flynn 1968; Lauterborn 1976), was used as a general indicator for stable cavitation activity. Broadband noise was used as an indicator for inertial cavitation activity (Chen et al. 2003). Analysis was performed on a small segment of the burst, $41 \mu \mathrm{s}$ in duration, beginning $14 \mu \mathrm{s}$ after the start of the burst. The time-domain hydrophone signal corresponding to each burst was converted to the frequency domain using a 2048-point Fast Fourier Transform (FFT). A Blackman-Harris window was used to reduce spectral leakage in the frequency domain, providing increased signal-to-noise ratio.

In the absence of cavitation, only the fundamental ultrasound drive signal and its harmonics were present, along with broadband electrical background noise (Fig. 2a). Broadband cavitation noise was manifested as an elevation in the signal amplitude between the harmonic peaks in the FFT magnitude spectrum (Fig. 2b). The broadband noise in a particular signal was quantified by integrating the "inter-peak" noise amplitude, $N A$, between 4 and $10 \mathrm{MHz}$. The relative noise enhancement, $R N E$, was calculated as the increase in noise amplitude relative to the average non-cavitating baseline noise amplitude, $\langle B N A>$, for 2100 recorded baseline bursts:

$$
R N E=\left[\frac{N A-\langle B N A\rangle}{\langle B N A\rangle}\right]
$$

For baseline signal collection, the catheter and drug lumens were inserted into a test tube filled with degassed ( $<36 \%$ of saturation), $0.2 \mu \mathrm{m}$ filtered water.

A true rise in broadband noise over baseline due to inertial cavitation was identified by setting a detection threshold for the system. The noise enhancement threshold, NET, was defined 
as the relative noise enhancement corresponding to 4 times the standard deviation of the baseline noise amplitude, $S D\{B N A\}$, for 2100 recorded baseline bursts:

$$
N E T=\left[\frac{4 \times S D\{B N A\}}{\langle B N A\rangle}\right]
$$

Each recorded snapshot was searched for instances in which the relative noise enhancement crossed the noise enhancement threshold, and the total number of ultrasound bursts containing inertial cavitation ("IC count") was tabulated. In addition, the maximum relative noise enhancement detected in a snapshot, $\max \{R N E\}$, compiled as an average from the 10 independent snapshots, was compared between treatment groups. Statistical analysis was performed on the $\max \{R N E\}$ data using One-Way ANOVA and Fisher's Least Significant Difference multiple comparison t-test at a 0.05 level of significance.

Subharmonic emission was identified by the presence of a subharmonic peak in the FFT magnitude spectrum at one half of the fundamental frequency (Fig. 2b). To quantify the subharmonic content in a recorded burst, the FFT magnitude spectrum was computed and the magnitude at the subharmonic frequency was taken as the subharmonic amplitude, $S A$. The relative subharmonic enhancement, $R S E$, was calculated as the increase in subharmonic amplitude relative to the average non-cavitating baseline subharmonic amplitude, $\langle B S A>$, for 2100 recorded baseline bursts:

$R S E=\left[\frac{S A-\langle B S A\rangle}{\langle B S A\rangle}\right]$

Processed in this way, each digitized burst yielded a single measure of the relative subharmonic enhancement, which was then plotted as a function of time over the $3.33 \mathrm{~s}$ duration of the snapshot. For a given snapshot, the subharmonic quantities were averaged over the 100 bursts to yield the average subharmonic enhancement, $<R S E>$. The average value of $\langle R S E>$ was compiled from the 10 independent snapshots and compared between treatment groups. Statistical 
analysis was performed on the $<R S E>$ data using One-Way ANOVA and Fisher's Least Significant Difference multiple comparison t-test at a 0.05 level of significance. 


\section{RESULTS}

Clot Lysis

The resulting clot weights and calculated lysis parameters for each treatment protocol are listed in Table 1. The One-Way ANOVA resulted in an overall p-value less than 0.001 , indicating that statistically significant differences exist among the mean clot weights. For all treatments with drug, clot weight was significantly reduced compared to the control $(\mathrm{p}<0.001)$. The combination of drug and ultrasound produced a significantly lower clot weight compared to the drug-only clot weight $(\mathrm{p}=0.004)$. The addition of Optison ${ }^{\mathrm{TM}}$ to the combined treatment significantly decreased clot weight further compared to the drug and ultrasound treatment $(\mathrm{p}=$ 0.006). No difference in mean clot weight was detected $(p=1.000)$ between the group in which Optison $^{\mathrm{TM}}$ was pre-exposed to ultrasound and the group in which Optison ${ }^{\mathrm{TM}}$ was not pre-exposed to ultrasound. Application of ultrasound and $\mathrm{Optison}^{\mathrm{TM}}$ in the absence of drug resulted in no significant difference in clot weight compared to the control $(\mathrm{p}=0.415)$.

Ultrasound exposure resulted in mild heating of the clot. For a catheter temperature maintained at $43^{\circ} \mathrm{C}$ within the water-filled polyimide sleeve, the steady-state clot temperature was found to be approximately $38^{\circ} \mathrm{C}$, as measured by a thermocouple inserted in the clot just above the catheter transducer.

\section{Cavitation}

Shown respectively in Figs. $\mathbf{3}$ and $\mathbf{4}$ are plots of relative noise enhancement and relative subharmonic enhancement versus time during the $3.33 \mathrm{~s}$ snapshot acquired at time zero in the 30 min ultrasound exposure. Each trace constitutes a point-by-point average of the 10 individual snapshots collected per treatment protocol. In the absence of $\operatorname{Optison}^{\mathrm{TM}}(D+U S), R N E$ and $R S E$ remained at baseline. When Optison ${ }^{\mathrm{TM}}$ was present, cavitation signals were highest and were identical for the two protocols in which Optison ${ }^{\mathrm{TM}}$ was not pre-exposed to ultrasound $(D+U S+O P$ and $U S+O P) . R N E$ rapidly increased to a peak value within $\sim 0.1 \mathrm{~s}$ and then fell back toward baseline, crossing below the detection threshold $(N E T=0.24)$ at $\sim 0.4 \mathrm{~s}$, and settling to 
baseline by $\sim 1 \mathrm{~s}$. Similarly, $R S E$ increased to a peak value within $\sim 0.1 \mathrm{~s}$. $R S E$, however, did not settle back to baseline, but instead remained at a slightly elevated value during the remainder of the snapshot. For the treatment in which Optison ${ }^{\mathrm{TM}}$ was pre-exposed to ultrasound $(D+U S+O P($ pre $))$, broadband noise and subharmonic activity were reduced compared to the other Optison $^{\mathrm{TM}}$ treatments: elevation in $R N E$ was observed in only the first ultrasound burst, which immediately dropped to baseline by the next burst; $R S E$ remained at a low, steady amplitude slightly above baseline.

The quantified cavitation signals per snapshot measured at various times throughout the 30 min exposure are shown in Fig. $5(\max \{R N E\})$ and Fig. $6(<R S E>)$. In the absence of Optison $^{\mathrm{TM}}(D+U S), \max \{R N E\}$ remained below the detection threshold throughout the $30 \mathrm{~min}$ exposure, and $<R S E>$ remained at baseline. For the snapshot at time zero, both $\max \{R N E\}$ and $<R S E>$ for $D+U S+O P$ and $U S+O P$ were significantly greater than $D+U S(\mathrm{p}<0.001)$. During the remainder of the 30 min exposure, and for all of the Optison ${ }^{\mathrm{TM}}$ protocols, $\max \{R N E\}$ showed no significant difference from $D+U S$, while $<R S E>$ was found to be significantly greater than $D+U S(\mathrm{p}<0.001)$.

Table 2 lists the total number of IC counts observed for the different treatment protocols. In accordance with the $\max \{R N E\}$ trends shown in Fig. 5, IC counts were only observed during the initial snapshot in the $30 \mathrm{~min}$ exposure, and the number of counts was greatest for the two protocols in which Optison ${ }^{\mathrm{TM}}$ was not pre-exposed to ultrasound $(D+U S+O P$ and $U S+O P)$. In the absence of Optison ${ }^{\mathrm{TM}}(D+U S)$ two IC counts were recorded. 


\section{DISCUSSION}

Ultrasound-accelerated, rt-PA-induced dissolution of plasma clot was performed in vitro using a catheter-mounted transducer in the presence and absence of Optison ${ }^{\mathrm{TM}}$. Cavitational mechanisms were investigated by monitoring acoustic signals emanating from the plasma clot, and quantifying subharmonic emission and broadband noise, indicative of stable and inertial cavitation respectively.

In the absence of Optison ${ }^{\mathrm{TM}}, 1.7 \mathrm{MHz}$ pulsed ultrasound significantly increased clot lysis in the presence of rt-PA. A previous study using the same in vitro model and exposure conditions showed that D-Dimer concentration increased proportional to the increased clot weight loss in the presence of ultrasound and rt-PA compared to rt-PA alone, suggesting enhanced enzymatic fibrinolysis rather than clot fragmentation (Soltani 2005). Thermal mechanisms are not believed to have played a role in the observed lysis enhancement. The minimal degree of heating observed in this and in similar experiments has been shown by Blinc et al. to be insufficient to account for the increased lysis (1993). Cavitation signals were either not detected (subharmonic amplitude remained at baseline) or were negligible (only two IC counts) in the corresponding radiated acoustic signal, suggesting that the observed lysis enhancement in the absence of contrast agent was due to non-cavitational mechanical effects of the ultrasound. This result is contrary to findings by Everbach and Francis (2000) who, using similar acoustic parameters, demonstrated in vitro by cavitation detection and static overpressure experiments a significant role of cavitation in ultrasound-accelerated fibrinolysis in the absence of contrast agent. The discrepancy may be due to differences in the content of cavitation nuclei or dissolved gas in the in vitro clot models used. The lack of cavitation observed in the clot of the current study is not surprising considering the cavitation threshold in blood has been measured to be 4.1 MPa by Deng et al. (1996), which is greater than the pressures used here. This result also corresponds with what would be expected for the given acoustic parameters in vivo, where naturally-occurring cavitation nuclei are scarce and cavitation thresholds may be even higher. 
When Optison ${ }^{\mathrm{TM}}$ was dispersed throughout the clot with rt-PA delivered, lysis enhancement doubled compared to the rt-PA and ultrasound exposure without Optison ${ }^{\mathrm{TM}}$, confirming the known synergistic effect of microbubbles and fibrinolytic agent (Cintas et al. 2004; Tachibana and Tachibana 1995). Unlike the previous studies demonstrating this effect, the in vitro model of the present study applied the contrast agent and fibrinolytic agent to the interior of the clot rather than in the fluid medium surrounding the clot. Inertial cavitation, observed as broadband noise in the acoustic signal, was present only at the start of the ultrasound exposure, and disappeared completely in less than one second. Subharmonic emission, an indicator for stable cavitation activity, was greatest at the start of the exposure, subsequently decreased in amplitude, yet persisted for the remainder of the 30 min treatment. The limited duration of inertial cavitation may be explained by liberated Optison ${ }^{\mathrm{TM}}$ yielding sub-resonant bubbles (i.e. bubbles smaller than resonance size at $1.7 \mathrm{MHz}$ ) that break up upon collapse, forming very small bubbles that are quickly driven to dissolution by the compressive force of surface tension. For a spherical air bubble in water, the resonance size is approximately $2 \mu \mathrm{m}$ radius at $1.7 \mathrm{MHz}$ (Leighton 1994). The long-lived subharmonic activity may be due to super-resonant bubbles that are too large to collapse inertially, yet are still capable of generating subharmonic emissions.

In the presence of Optison ${ }^{\mathrm{TM}}$ alone, ultrasound exposure generated the same cavitation activity observed with rt-PA plus Optison ${ }^{\mathrm{TM}}$, but resulted in no significant clot weight loss. This result suggests that mechanical disruption (i.e. fragmentation) of the clot was not a contributing mechanism at the exposure parameters employed, and that the increased lysis observed in the presence of Optison $^{\mathrm{TM}}$ and rt-PA was due to increased enzymatic fibrinolysis. The lack of effect of microbubbles in the absence of lytic agent, also observed by Tachibana and Tachibana (1995), is not surprising in the current study because of the limited duration of inertial cavitation observed. Other ultrasound thrombolysis studies (Cintas et al. 2002; Culp et al. 2003, 2004) have demonstrated clot dissolution due to contrast agents alone using acoustic parameters similar to those in the current study. A possible explanation for the different results is that in these studies 
contrast agent was continually replenished, either by multiple injections or by re-circulating bubbles past the clot. Replenishment of microbubbles, or a change in acoustic parameters, such as acoustic pressure amplitude, pulse length, or duty cycle, may increase the persistence and quantity of inertial cavitation, possibly to an extent where mechanical effects alone become important.

For the treatment in which Optison ${ }^{\mathrm{TM}}$ was pre-exposed to ultrasound before drug delivery, the cavitation activity that occurred at the start of rt-PA treatment was significantly reduced compared to the standard protocol with Optison ${ }^{\mathrm{TM}}$. Inertial cavitation was effectively eliminated: only the first ultrasound burst contained broadband noise. A possible explanation for this result is that all of the candidate sub-resonant bubbles were depleted in the pre-exposure and thus were not available to nucleate inertial cavitation when the drug was present. The lowamplitude subharmonic emission, however, was still present during the 30 min exposure and at the same magnitude as in the other protocols with Optison ${ }^{\mathrm{TM}}$. This result supports the notion of super-resonant bubbles: large bubbles (which are less influenced by surface tension) do not dissolve as quickly, and could be held in place by the dense fibrin matrix of the clot. The resulting lysis enhancement was the same as that obtained for the treatment in which Optison ${ }^{\mathrm{TM}}$ was not pre-exposed to ultrasound. The fact that the 30 min subharmonic emission was common to both protocols suggests a possible correlation between the increased lysis and the subharmonic emission. The brief inertial cavitation activity that occurred at the beginning of the exposure appears to be inconsequential to the observed lysis since its presence or absence with respect to rt-PA treatment did not affect the resulting clot weight. Additional treatment protocols, however, are required to confirm this correlation to the subharmonic. Using lower peak negative pressures to separate stable from inertial cavitation, for example, would be an ideal next step for this work.

A possible alternative explanation is that the inertial cavitation produced microfragmentation or perforation of the clot. Such small-scale mechanical disruption by itself (i.e. in the absence of rt-PA) may be too small to measure as a reduction in clot weight, but could 
potentially expose greater clot surface area to the rt-PA which could increase the overall lysis. Although the Optison ${ }^{\mathrm{TM}}$ pretreatment temporally separated the inertial cavitation from the rt-PA treatment, any permanent effect of the cavitation that occurred during the ultrasound preexposure, such as clot micro-fragmentation, could carry over to and influence the rt-PA treatment. Based on the results of the current test alone, this possibility cannot be ruled out as a potential mechanism for the increased lysis.

In support of the proposed correlation between the subharmonic emission and the increased lysis achieved in the presence of Optison ${ }^{\mathrm{TM}}$, a recent study by Datta et al. (2006) identified an important role of stable cavitation in the enhancement of thrombolysis. After measuring cavitation thresholds in clot, they exposed in vitro clots to $120 \mathrm{kHz}$ ultrasound using separate treatment regimes to investigate the effects of stable and inertial cavitation. They found that t-PA mediated thrombolysis was not only significantly enhanced in the presence of stable cavitation, but was greater in the presence of stable cavitation alone compared to both stable and inertial cavitation present.

Mechanisms related to stable cavitation, such as steady microstreaming, could enhance fibrinolysis by promoting local mass transfer and thus the rate of penetration of the drug into the clot matrix. In the present study, the subharmonic emission was used as a general indicator for stable cavitation. The suggested correlation to the subharmonic emission does not single out mechanisms specifically related to the generation of a subharmonic. Other types of stable bubble activity, including oscillations that occur below the pressure threshold for subharmonic emission, can occur simultaneously and may also be important. However, it is well known that surface waves on the bubble wall, which have been associated with subharmonic emissions (Phelps 1996), are effective in generating microstreaming flows around bubbles undergoing repetitive large amplitude motion (Elder 1958). Subharmonic emissions may serve as a sufficient, if not a necessary, condition for microbubble-enhanced ultrasound-accelerated fibrinolysis. 
If stable cavitation alone is capable of significantly enhancing rt-PA-mediated thrombolysis, the clinical relevance is that one could potentially avoid the safety risks associated with inertial cavitation (i.e. tissue hemorrhage, hemolysis), yet still enjoy the lysis-enhancing effect of microbubbles by choosing ultrasound exposure parameters to promote stable cavitation while minimizing inertial cavitation. A judicious choice of ultrasound exposure parameters, coupled with Optison $^{\mathrm{TM}}$ to serve as the initial source of nuclei, could serve to maximize stable cavitation activity over the duration of the exposure and the concomitant fibrinolytic effects. 


\section{CONCLUSION}

Cavitation detection was performed in vitro to investigate mechanisms of $1.7 \mathrm{MHz}$ ultrasound-accelerated rt-PA-mediated clot dissolution in the presence and absence of Optison ${ }^{\mathrm{TM}}$. In the absence of contrast agent, ultrasound plus rt-PA resulted in $45 \pm 19 \%$ lysis enhancement relative to rt-PA alone. We conclude that the ultrasound accelerated rt-PA-induced fibrinolysis through non-cavitational, non-thermal mechanisms, as indicated by the lack of cavitation signals in the radiated acoustic spectrum, and the minimal heating observed. Addition of Optison ${ }^{\mathrm{TM}}$ increased lysis enhancement to $88 \pm 25 \%$. Both stable and inertial cavitation signals were detected. Clot fragmentation, a possible consequence of inertial cavitation, is not believed to have contributed to the increased lysis since Optison ${ }^{\mathrm{TM}}$ without rt-PA caused no reduction in clot weight. When Optison ${ }^{\mathrm{TM}}$ in the clot was pretreated with ultrasound to remove inertial cavitation but preserve subharmonic emission during the rt-PA treatment, lysis was the same as for the standard treatment with Optison ${ }^{\mathrm{TM}}$, suggesting a possible correlation between the increased lysis and the presence of the subharmonic emission. This result indicates a potentially important role of stable cavitation in microbubble-enhanced ultrasound-accelerated rt-PA-mediated thrombolysis. Mechanisms related to stable cavitation, such as steady microstreaming, could enhance lysis by promoting local mass transfer and thus the rate of penetration of the drug into the clot matrix. 


\section{REFERENCES}

Blinc A, Francis C, Trudnowski J, Carstensen E. Characterization of ultrasound-potentiated fibrinolysis in vitro. Blood 1993;81:2636-2643.

Carr M, Hardin C. Fibrin has larger pores when formed in the presence of erythrocytes. Am J Physiol 1987;253:H1069-H1073.

Cintas P, Le Traon AP, Larrue V. High Rate of Recanalization of Middle Cerebral Artery Occlusion During 2-MHz Transcranial Color-Coded Doppler Continuous Monitoring Without Thrombolytic Drug. Stroke 2002;33:626-628.

Cintas P, Nguyen F, Boneu B, Larrue V. Enhancement of enzymatic fibrinolysis with 2-MHz ultrasound and microbubbles. J Thromb Haemost 2004;2:1163-1166.

Culp W, Erdem E, Roberson P, Husain M. Microbubble Potentiated Ultrasound as a Method of Stroke Therapy in a Pig Model: Preliminary Findings. J Vascular Interv Rad 2003;14:14331436.

Culp W, Porter T, Lowery J, et al. Intracranial Clot Lysis With Intravenous Microbubbles and Transcranial Ultrasound in Swine. Stroke 2004;35:2407-2411.

Datta S, Coussios C, McAdory L, et al. Correlation of Cavitation with Ultrasound Enhancement of Thrombolysis. Ultrasound Med Biol 2006;32:1257-1267.

Deng C, Xu Q, Apfel R, Holland C. In vitro measurements of inertial cavitation thresholds in human blood. Ultrasound Med Biol 1996;22:939-948.

Elder S. Cavitation Microstreaming. J Acoust Soc Am 1958;31:54-64.

Eller A and Flynn H. Generation of Subharmonics of Order One-Half by Bubbles in Sound Field. J Acoust Soc Am 1968;46:722-727.

Everbach E, Francis C. Cavitational Mechanisms In Ultrasound-Accelerated Thrombolysis At 1 MHz. Ultrasound Med Biol 2000;26:1153-1160.

Francis C, Onundarson P, Carstensen E, et al. Enhancement of fibrinolysis in vitro by ultrasound. J Clin Invest 1992;90:2063-2068. 
Francis C, Blinc A, Lee S, Cox C. Ultrasound accelerates transport of recombinant tissue plasminogen activator into clots. Ultrasound Med Biol 1995;21:419-424.

Harpaz D, Chen X, Francis C, Marder V, Meltzer R. Ultrasound enhancement of thrombolysis and reperfusion in vitro. JACC 1993;21:1507-1511.

IEC. IEC 60601-2-37-Medical electrical equipment-Part 2-37: Particular requirements for the safety of ultrasonic medical diagnostic and monitoring equipment. Geneva: International Electrotechnical Commission, 2004.

Kashyap A, Blinc A, Marder V, Penney D, Francis C. Acceleration of fibrinolysis by ultrasound in a rabbit ear model of small vessel injury. Thromb Res 1994;76:475-485.

Kornowski R, Meltzer R, Chernine A, Vered Z, Battler A. Does external ultrasound accelerate thrombolysis? Results from a rabbit model. Circulation 1994;89:339-344.

Lauer C, Burge R, Tang D, et al. Effect of ultrasound on tissue-type plasminogen activatorinduced thrombolysis. Circulation 1992;86:1257-1264.

Lauterborn W. Numerical investigation of nonlinear oscillations of gas bubbles in liquids. $\mathrm{J}$ Acoust Soc Am 1976;59:283-293.

Leighton T. The Acoustic Bubble. London: Academic Press, 1994.

Mizushige K, Kondo I, Ohmori K, Hirao K, Matsuo H. Enhancement of ultrasound-accelerated thrombolysis by echo contrast agents: dependence on microbubble structure. Ultrasound Med Biol 1999;25:1431-1437.

Phelps A, Leighton T. High-resolution bubble sizing through detection of the subharmonic response with a two-frequency excitation technique. J Acoust Soc Am 1996;99:1985-1992.

Podell S, Burrascano C, Gaal M, Golec B, Maniquis J, Mehlhaff P. Physical and biochemical stability of Optison ${ }^{\circledR}$, an injectable ultrasound contrast agent. Biotechnol Appl Biochem 1999;30:213-223.

Riggs P, Francis C, Bartos S, Penney D. Ultrasound enhancement of rabbit femoral artery thrombolysis. Cardiovas Surg 1997;5:201-207. 
Shlansky-Goldberg R, Cines D, Sehgal C. Catheter-delivered Ultrasound Potentiates in Vitro Thrombolysis. JVIR 1996; 7:313-320.

Soltani A. The Safety of Using High Frequency, Low Intensity Ultrasound to Enhance Thrombolysis. ISTU 2005 Conference Proceedings.

Tachibana K and Tachibana S. Albumin Microbubble Echo-Contrast Material as an Enhancer for Ultrasound Accelerated Thrombolysis. Circulation 1995;92:1148-1150.

Tachibana K and Tachibana S. Prototype Therapeutic Ultrasound Emitting Catheter for Accelerating Thrombolysis. J Ultrasound Med 1997;16:529-535.

Wu Y, Unger E, McCreery T, et al. Binding and lysing of blood clots using MRX-408. Invest Radiol 1998;33:880-885.

Yang X, Church C. A model for the dynamics of gas bubbles in soft tissue. J Acoust Soc Am 2005;118:3595-3606. 


\section{TABLE HEADINGS}

Table 1. Clot weight, percent lysis, and percent lysis enhancement (all AVG $\pm \mathrm{SD}$ ) obtained for each treatment protocol. P-values from statistical comparisons between groups are shown.

Table 2. The total number of IC counts detected within the initial $3.33 \mathrm{~s}$ snapshot ( $0 \mathrm{~min})$ and collectively within the snapshots obtained during the remainder of the 30 min exposure $(1-30$ min). An IC count was defined as a burst value of relative noise enhancement, $R N E$, greater than the noise enhancement threshold, $N E T=0.24$. 
Table 1

\begin{tabular}{|c|c|c|c|c|c|c|c|c|}
\hline \multirow{2}{*}{$\begin{array}{c}\text { Treatment } \\
\text { Protocol }\end{array}$} & \multirow{2}{*}{$\begin{array}{c}\text { Clot Wt. } \\
\text { (mg) }\end{array}$} & \multirow{2}{*}{$\begin{array}{c}\text { Clot Lysis } \\
(\%)\end{array}$} & \multirow{2}{*}{$\begin{array}{c}\text { Lysis } \\
\text { Enhancement } \\
(\%)\end{array}$} & \multicolumn{5}{|c|}{ Statistical Comparison vs. } \\
\hline & & & & U & 0 & $\mathscr{O}$ & ติ & 2 \\
\hline $\mathrm{C}$ & $19.01 \pm 0.45$ & $0.00 \pm 1.06$ & $\mathrm{~N} / \mathrm{A}$ & & & & & 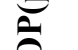 \\
\hline $\mathrm{D}$ & $17.55 \pm 0.55$ & $7.68 \pm 1.14$ & N/A & .000 & & & 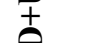 & 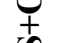 \\
\hline $\mathrm{D}+\mathrm{US}$ & $16.90 \pm 0.30$ & $11.10 \pm 0.83$ & $44.52 \pm 18.79$ & .000 & .004 & & & \\
\hline $\mathrm{D}+\mathrm{US}+\mathrm{OP}$ & $16.27 \pm 0.34$ & $14.41 \pm 0.85$ & $87.67 \pm 24.89$ & .000 & .000 & .006 & & \\
\hline $\mathrm{D}+\mathrm{US}+\mathrm{OP}($ pre $)$ & $16.27 \pm 0.54$ & $14.41 \pm 1.11$ & $87.67 \pm 26.53$ & .000 & .000 & .006 & 1.000 & \\
\hline US+OP & $18.83 \pm 0.67$ & $0.95 \pm 1.33$ & N/A & .415 & .000 & .000 & .000 & .000 \\
\hline
\end{tabular}




\section{Table 2}

\begin{tabular}{|c|c|c|c|c|c|}
\hline \multicolumn{2}{|c|}{} & D+US & $\begin{array}{c}\text { D+US } \\
+ \text { OP }\end{array}$ & $\begin{array}{c}\text { D+US } \\
+ \text { OP(pre) }\end{array}$ & US+OP \\
\hline $\begin{array}{c}\text { Total Number of } \\
\text { Bursts Analyzed }\end{array}$ & 0 min & 1000 & 1000 & 1000 & 1000 \\
\cline { 2 - 6 } & $1-30 \mathrm{~min}$ & 6000 & 6000 & 6000 & 6000 \\
\hline $\begin{array}{c}\text { Total Number of } \\
\text { IC Counts }\end{array}$ & $0 \mathrm{~min}$ & 2 & 103 & 5 & 111 \\
\cline { 2 - 6 } & $1-30 \mathrm{~min}$ & 0 & 0 & 0 & 0 \\
\hline
\end{tabular}




\section{FIGURE CAPTIONS}

Fig. 1. Diagram of experimental setup. (a) Side view of test tube. (b) Top view of test tube.

Fig. 2. FFT amplitude spectrum of acoustic signal measured (a) in the absence of cavitation (baseline signal), and (b) in the presence of both stable and inertial cavitation, as indicated by a subharmonic peak at $0.85 \mathrm{MHz}$ and broadband noise elevation.

Fig. 3. Relative noise enhancement versus time during a $3.33 \mathrm{~s}$ snapshot obtained at time zero in the 30 min ultrasound exposure. Each trace is an average over $\mathrm{N}=10$ snapshots. $D+U S$ shows no noise enhancement. $D+U S+O P$ and $U S+O P$ show identical noise enhancement rise and subsequent fall back to baseline. $D+U S+O P($ pre $)$ shows noise enhancement only in the first captured ultrasound burst.

Fig. 4. Relative subharmonic enhancement versus time during a $3.33 \mathrm{~s}$ snapshot obtained at time zero in the 30 min ultrasound exposure. Each trace is an average over $\mathrm{N}=10$ snapshots. $D+U S$ shows no subharmonic enhancement. $D+U S+O P$ and $U S+O P$ show identical, high-amplitude subharmonic enhancement, while $D+U S+O P$ (pre) shows low-amplitude subharmonic enhancement.

Fig. 5. (a) Maximum noise enhancement per snapshot graphed as a function of ultrasound exposure time. Each point corresponds to an average of $\mathrm{N}=10$ snapshots. (b) Bar graph comparison of $\max \{R N E\}$ for the snapshot taken at time zero $(0 \mathrm{~min})$ and the average $\max \{R N E\}$ for snapshots obtained during the remainder of the $30 \mathrm{~min}$ exposure. Error bars indicate standard deviation. 
Fig. 6. (a) Average subharmonic enhancement per snapshot graphed as a function of ultrasound exposure time. Each point corresponds to an average of $\mathrm{N}=10$ snapshots. (b) Bar graph comparison of $<R S E>$ for the snapshot taken at time zero and the average $<R S E>$ for snapshots obtained during the remainder of the 30 min exposure. Error bars indicate standard deviation. 
Fig. 1

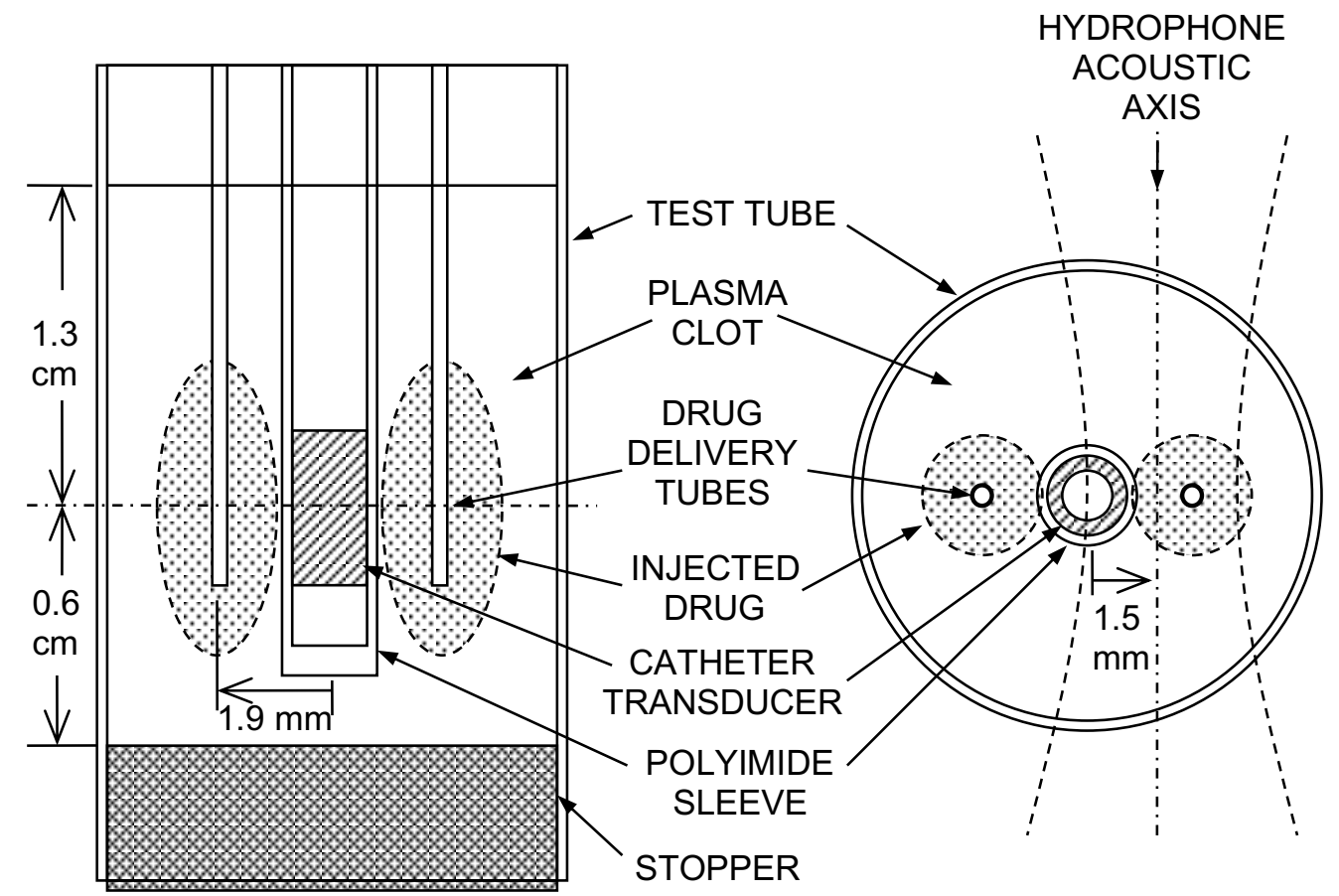

(a)

(b) 
Fig. 2a

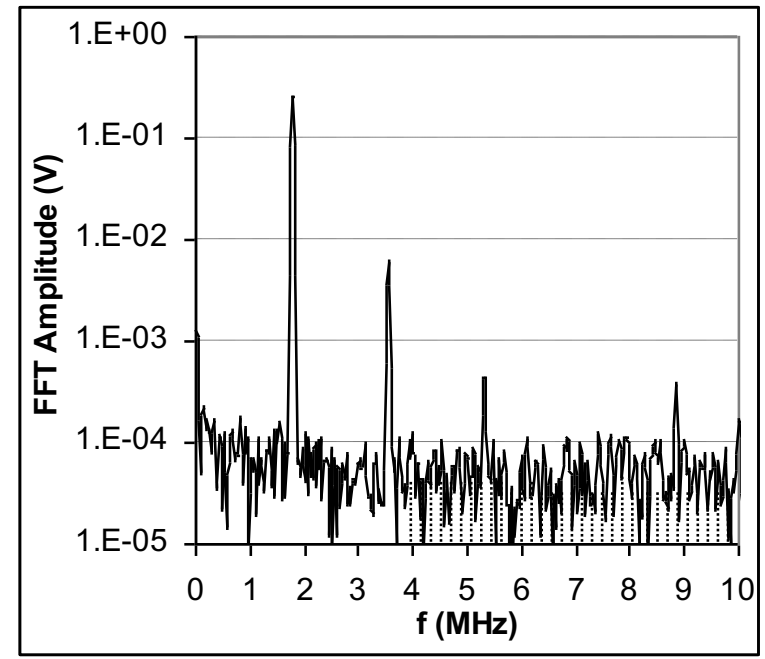


Fig. 2b

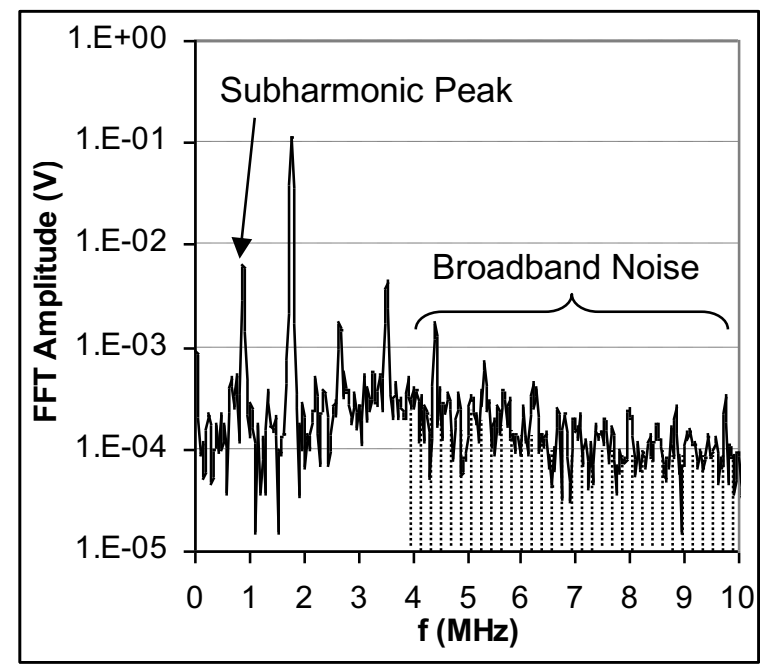


Fig. 3

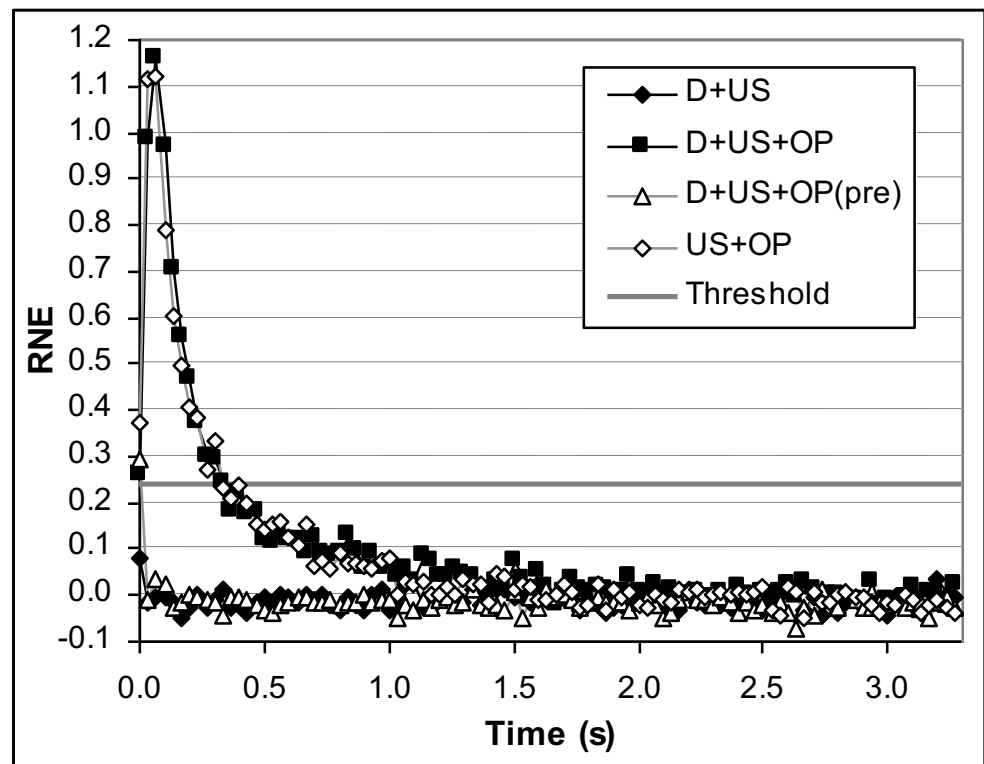


Fig. 4

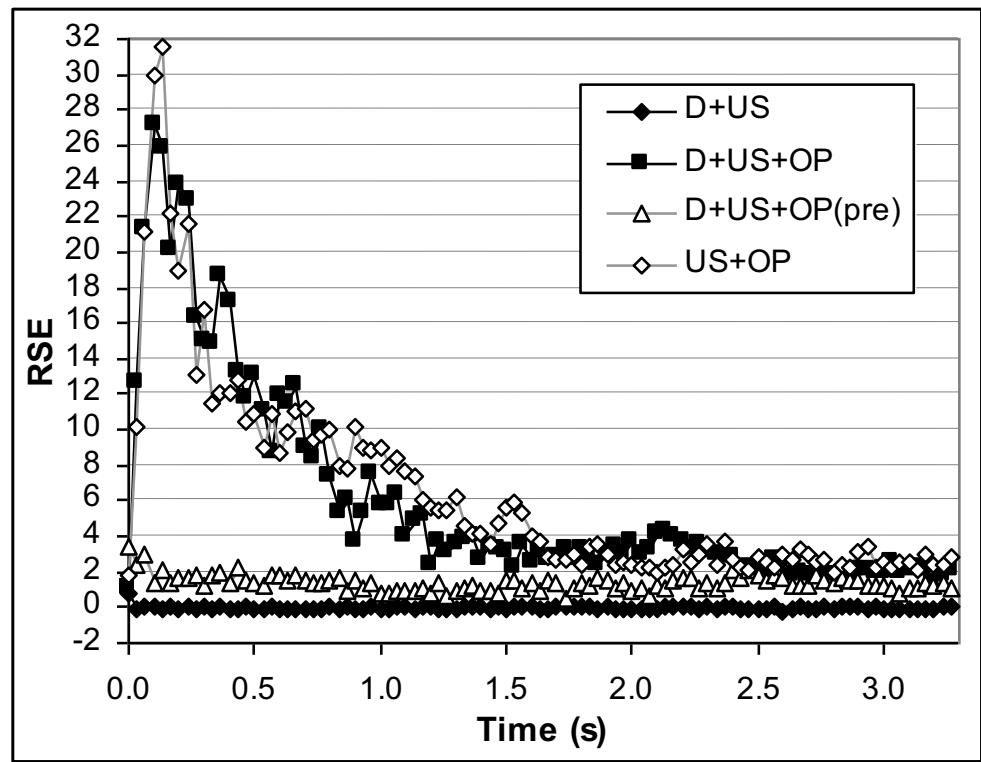


Fig. 5a

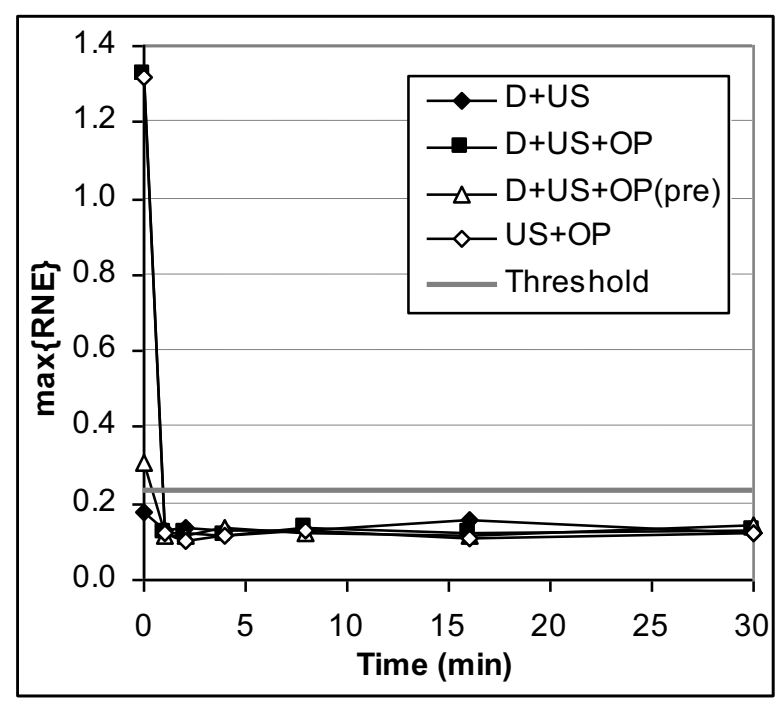


Fig. 5b

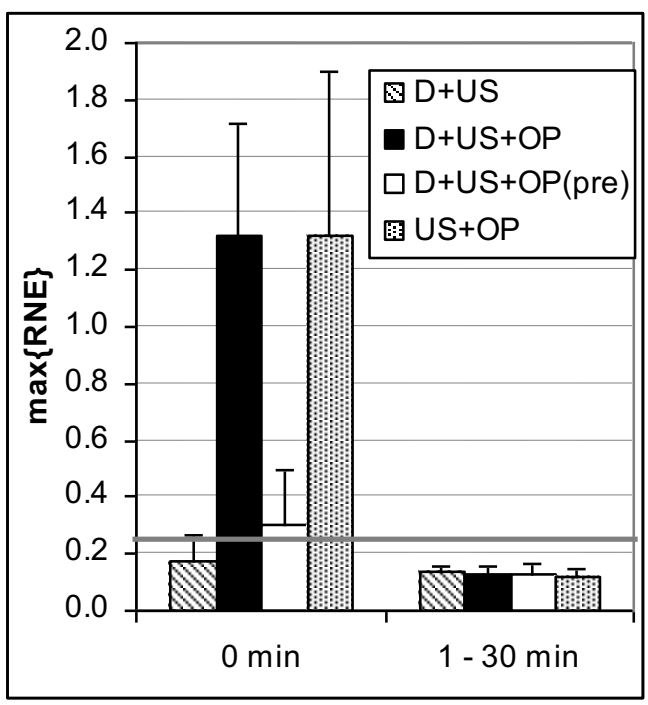


Fig. 6a

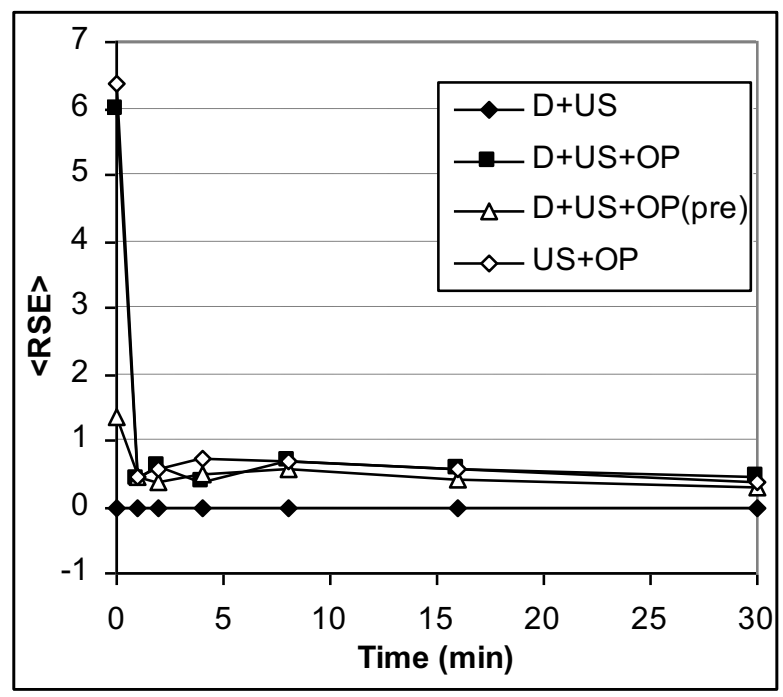


Fig. 6b

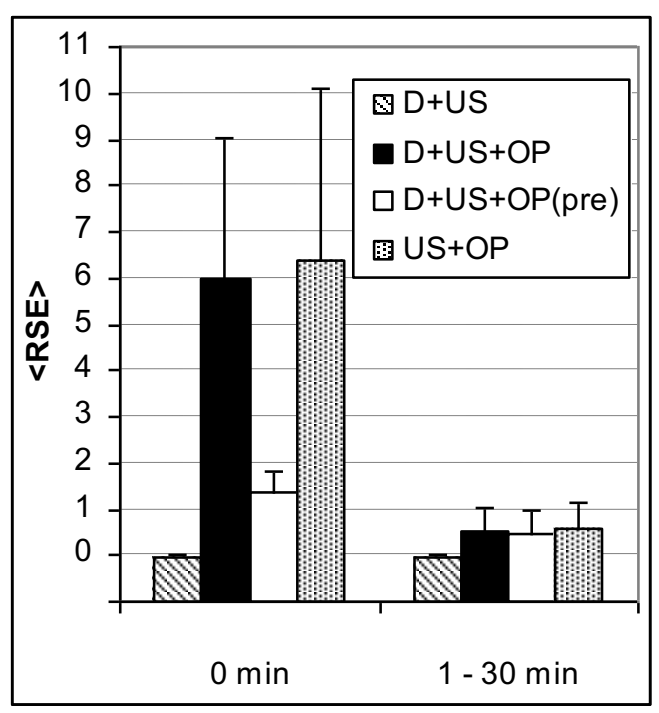

